\title{
Gut microbiota of humans, dogs and cats: current knowledge and future opportunities and challenges
}

\author{
Ping Deng ${ }^{1}$ and Kelly S. Swanson ${ }^{1,2,3 *}$ \\ ${ }^{1}$ Department of Animal Sciences, University of Illinois, 162 Animal Sciences Laboratory, 1207 West Gregory Drive, Urbana, \\ IL 61801, USA \\ ${ }^{2}$ Department of Veterinary Clinical Medicine, University of Illinois, 162 Animal Sciences Laboratory, 1207 West Gregory \\ Drive, Urbana, IL 61801, USA \\ ${ }^{3}$ Division of Nutritional Sciences, University of Illinois, 162 Animal Sciences Laboratory, 1207 West Gregory Drive, Urbana, \\ IL 61801, USA
}

(Submitted 22 November 2013 - Final revision received 14 July 2014 - Accepted 22 July 2014 - First published online 21 November 2014 )

\begin{abstract}
High-throughput DNA sequencing techniques allow for the identification and characterisation of microbes and their genes (microbiome). Using these new techniques, microbial populations in several niches of the human body, including the oral and nasal cavities, skin, urogenital tract and gastrointestinal tract, have been described recently. Very little data on the microbiome of companion animals exist, and most of the data have been derived from the analysis of the faeces of healthy laboratory animals. High-throughput assays provide opportunities to study the complex and dense populations of the gut microbiota, including bacteria, archaea, fungi, protozoa and viruses. Our laboratory and others have recently described the predominant microbial taxa and genes of healthy dogs and cats and how these respond to dietary interventions. In general, faecal microbial phylogeny (e.g. predominance of Firmicutes, Bacteroidetes, Proteobacteria and Actinobacteria) and functional capacity (e.g. major functional groups related to carbohydrate, protein, DNA and vitamin metabolism; virulence factors; and cell wall and capsule) of the canine and feline gut are similar to those of the human gut. Initial sequencing projects have provided a glimpse of the microbial super-organism that exists within the canine and feline gut, but leaves much to be explored and discovered. As DNA provides information only about potential functions, studies that focus on the microbial transcriptome, metabolite profiles, and how microbiome changes affect host physiology and health are clearly required. Future studies must determine how diet composition, antibiotics and other drug therapies, breed and disease affect or are affected by the gut microbiome and how this information may be used to improve diets, identify disease biomarkers and develop targeted disease therapies.
\end{abstract}

\section{Key words: Microbiomes: Health: Canine: Feline}

Microbes, as the smallest forms of life that exist in every corner of the world, including the animal body, have enormous impacts on their host. The gastrointestinal tract (GIT) is the primary microbial habitat in humans and other animal species. In the past, knowledge pertaining to gastrointestinal microbiology and the impact of microbiota on host health was largely obtained using traditional culture techniques. As less than $1 \%$ of the microbes in the body can be cultured and studied in the laboratory, progress in this filed has greatly been hindered by the lack of appropriate research approaches. The recent availability of high-throughput DNA sequencing techniques coupled with new bioinformatic developments that allow for the identification and characterisation of microbes and their genes (microbiome) in and on the body has dramatically changed the research landscape.

When studying microbial phylogenetic structure, the primary target has most commonly been the small subunit ribosomal RNA (16S rRNA) gene. This gene is ubiquitously

\footnotetext{
Abbreviations: DMB, DM basis; FISH, fluorescent in situ hybridisation; FOS, fructo-oligosaccharides; GIT, gastrointestinal tract; GOS, galactooligosaccharide; HMP, Human Microbiome Project; HP, high protein; IBD, inflammatory bowel disease; MetaHIT, Metagenomics of the Human Intestinal Tract; MPMC, moderate-protein, moderate-carbohydrate; PDX, polydextrose; RFLP, restriction fragment length polymorphism; rRNA, ribosomal RNA; SCF, soluble corn fibre.

*Corresponding author: K. S. Swanson, fax +1 217333 7861, email ksswanso@illinois.edu

This article was published as part of the WALTHAM International Nutritional Sciences Symposium Proceedings 2013.

This paper was published as part of a supplement to British Journal of Nutrition, publication of which was supported by an unrestricted educational grant from Mars Incorporated. The papers included in this supplement were invited by the Guest Editor and have undergone the standard journal formal review process. They may be cited.
} 
present in all bacteria and archaea and contains both conserved and variable sequence regions, enabling researchers to distinguish organisms at different phylogenetic levels. Several molecular methods based on the microbial 16S rRNA gene have emerged and have been used as tools to identify and quantify microbes, including quantitative $\mathrm{PCR}^{(1)}$; fluorescent in situ hybridisation $(\mathrm{FISH})^{(2)}$; gel-based techniques such as restriction fragment length polymorphism (RFLP) analysis ${ }^{(3)}$, denaturing gradient gel electrophoresis ${ }^{(4)}$, and temperature gradient gel electrophoresis ${ }^{(5)}$; and DNA sequencing techniques ${ }^{(6)}$ such as Sanger sequencing, Illumina sequencing (Illumina, Inc.) and 454-pyrosequencing sequencing (Roche Applied Science). The next-generation sequencing technologies (e.g. 454; Illumina) generate shorter sequences compared with the traditional Sanger sequencing method, but are much faster and cheaper. Both methodologies are continuing to improve rapidly, providing progressively greater sequence length and depth. Currently, the MiSeq Illumina platform is considerably cheaper than 454-pyrosequencing sequencing, providing even more sequence depth per dollar ${ }^{(7)}$. Over the past few years, it has become clear that studying the entire microbiome rather than phylogeny alone is essential to truly understand its role in the host. Thus, shotgun sequencing that analyses all DNA in a sample provides information about the functional capacity of the microbial genome. With these and other high-throughput sequencing techniques being developed, the bottleneck of such studies is clearly computational capacity and biological interpretation by investigators.

International projects focused on the human microbiome, including the Human Microbiome Project (HMP; http://www. hmpdacc.org) and the Metagenomics of the Human Intestinal Tract (MetaHIT; http://www.metahit.eu), have sampled and described the microbiota of several niches, including the oral cavity, nasal cavity, skin, urogenital tract and GIT. In humans, diseases associated with microbial dysbiosis include obesity ${ }^{(8,9)}$, diabetes $^{(10,11)}$, inflammatory bowel disease $(\mathrm{IBD})^{(12,13)}$ and allergies $^{(14)}$. Compared with the human microbiome data, very little canine and feline microbiome data exist, however, with most of the data being derived from the analysis of the faeces of healthy laboratory animals.

Compared with humans and domestic livestock species, dogs and cats have a relatively simple GIT and do not rely on the microbiota for energy. Nonetheless, recent studies have revealed that the feline and canine GIT microbial community is composed of several hundred microbial phylotypes ${ }^{(15,16)}$. The fundamental purpose of studying canine and feline microbiota is to gain a better understanding of the taxonomy, dynamics, and functions of microbial communities, the relationships between microbial members, the substances produced and consumed by microbes, the influence of environmental factors (e.g. diet, drugs and housing environment), the interaction with the host, and the differences between healthy and diseased populations. In addition to animal health implications, companion animal microbiota research may have relevance to human health, given the exchange of microbes between humans and their pets. It has been shown that dog owners share more microbiota with their own dogs than with other dogs, with stronger effects of cohabitation on the skin microbiota than on the oral or faecal microbiota ${ }^{(17)}$. Moreover, enterococci from the intestinal microbiota of cats and dogs may act as a reservoir of resistance genes for human pathogens ${ }^{(18)}$. Despite the progress made over the past decade, this field is filled with challenges and opportunities. This review briefly summarises and compares the microbiome studies recently carried out in human subjects, dogs and cats and highlights some of the opportunities and challenges in the field.

\section{Human microbiome}

Gastrointestinal microbes play a fundamental role in human health and disease, participating in energy harvest and storage $^{(19)}$, development of the immune system ${ }^{(20,21)}$ and many other processes. Newfound knowledge regarding the human microbiota has mainly come from large international microbiome projects such as the HMP and the MetaHIT project. The US National Institutes of Health-funded HMP has provided financial support for large interdisciplinary teams of scientific experts to study human microbiota and hostmicrobe relationships. The 5 -year project, which provided $\$ 150$ million over that period ${ }^{(22)}$, focused on producing reference genomes (viral, bacterial and eukaryotic) and establishing a population-scale framework for subsequent metagenomic analysis and microbial community structure and function studies ${ }^{(23)}$. The MetaHIT, a 22-million euro project funded by the European Commission, also focused on investigating the gut microbiome using the whole-genome shotgun technology in a wide range of healthy individuals and patients (http://www.metahit.eu). The ultimate objective of these projects was to demonstrate the role of microbiota in the maintenance of health and prevention of disease. These large multifaceted research initiatives have laid a foundation for future microbiome studies.

The initial microbiome projects characterised the ecology of the human microbial community from a large cohort and a set of distinct body habitats and revealed that even healthy individuals differ remarkably with regard to the microbes that occupy the oral cavity, skin, vagina and gut ${ }^{(24)}$. Intraindividual variation was found to be consistently lower than inter-individual variation over time in both phylogeny and metabolic functions ${ }^{(24)}$. It was demonstrated that oral and stool microbial communities were especially diverse in terms of community membership, while vaginal sites harboured particularly simple communities ${ }^{(24)}$. Comparison of skin, urogenital and oral bacterial sequences with near full-length sequences present in the large intestines and faeces of healthy individuals revealed that distinct sites of human body harbour distinct sets of bacteria ${ }^{(24)}$. Recent reviews have summarised the research to date on the skin ${ }^{(25,26)}$ and oral microbiome ${ }^{(27)}$. These large-scale studies used 16S rRNA sequencing in healthy adults and revealed that skin bacterial diversity largely depends on the topographical location on the body, and most of the skin bacteria are categorised into four different phyla: Actinobacteria; Firmicutes; Proteobacteria; Bacteroidetes. Furthermore, it was demonstrated that specific bacteria 
were associated with moist, dry and sebaceous microenvironments ${ }^{(25,26)}$. Moreover, the human mouth was shown to harbour about 1000 bacterial species colonised in distinct sites, with representatives from the phyla Actinobacteria, Bacteroidetes, Firmicutes, Proteobacteria, Spirochaetes, Synergistetes, and Tenericutes and the uncultured divisions GN02, SR1 and TM7 being shown to be present using culture-independent methods ${ }^{(27)}$. In a study carried out in 120 individuals from twelve worldwide locations, no significant geographical differences were found in the salivary microbiota, indicating that diet and environment may not significantly influence the composition of the oral microbiome ${ }^{(28)}$.

The human gut environment is remarkably different in each anatomical region in terms of physiology, transit time, substrate availability, host secretions, $\mathrm{pH}$ and oxygen tension ${ }^{(29)}$. Therefore, the microbial communities in the proximal and distal gastrointestinal regions can be remarkably diverse. Unfortunately, most of the available microbiome data published thus far have been derived from the analysis of faecal samples, mainly representing microbiota from the lumen of the distal large intestine ${ }^{(29)}$. Studies focused on the phylogenetic composition of the healthy human gut have demonstrated that Firmicutes, Bacteroidetes and Actinobacteria constitute the majority of the dominant bacterial phyla, with smaller proportions of Proteobacteria and Verrucomicrobia ${ }^{(24,30-33)}$.

Gut bacteria are involved in metabolite production and interact with the host in a carefully orchestrated manner to achieve metabolic communication, which has been reviewed by Nicholson et al. ${ }^{(34)}$. For example, Clostridial clusters IV and XIVa of the Firmicutes phylum produce SCFA that decrease colonic $\mathrm{pH}$, inhibit the growth of pathogens, stimulate water and $\mathrm{Na}$ absorption, participate in cholesterol synthesis and provide energy to the colonic epithelial cells $^{(35,36)}$. Other bacteria, including Lactobacillus, Bifidobacterium, Enterobacter, Bacteroides and Clostridium, have been proven to be involved in bile acid metabolism and to affect the absorption of dietary fats and lipid-soluble vitamins ${ }^{(37,38)}$. Faecalibacterium prausnitzii and Bifidobacterium have been shown to be associated with choline metabolism to modulate lipid metabolism and glucose homeostasis ${ }^{(39,40)}$ Integrating current sequencing techniques and metagenomic data with high-throughput metabolomic analytical platforms allows the investigation of global metabolite profiles of microbial organisms that may offer another strategy for studying the link between microbiota and host metabolism.

As part of the MetaHIT project, Qin et al. ${ }^{(41)}$ used Illuminabased metagenomic sequencing to characterise the human gut microbial genes using faecal samples of 124 European individuals. They generated $576.7 \mathrm{~Gb}$ of sequence, almost 200 times greater than that in all previous studies, assembled it into contigs, predicted 3.3 million unique open reading frames and explored the existence of a common set of fifty-seven microbial species in the cohort. They identified functional groups essential for all bacteria and a few that are potentially specific to those residing in the gut. Several metabolic pathways (e.g. central carbon metabolism and amino acid synthesis) and important protein complexes (e.g. RNA and DNA polymerases, ATP synthase and general secretory apparatus) appear to be essential for all microbes. Putative gut-specific functions included those involved in host proteins (e.g. collagen, fibrinogen and fibronectin) and in harvesting sugars of the globoseries glycolipids. This metagenomic study provided a broad view of the functions important for bacterial life in the gut and indicated that many bacterial species are shared by different individuals. Challenges still remain, with increasing needs in terms of the computational capacity and biological interpretation and application to human health. These assays and concepts, however, have taken us one step closer to understanding how one may maintain a healthy gut by limiting the growth and activity of pathogenic bacteria, while promoting those that are deemed beneficial.

Complex interactions between the environment, host genetics and microbiome maintain gut homeostasis. Additional projects supported by the HMP have investigated the association between specific components and dynamics of the microbiome in various disease conditions. Disturbances in the microbiota may be early warning signs for certain diseases. Turnbaugh et $a l .{ }^{(8)}$ conducted one of the first studies to demonstrate how the gut microbiome contributes to obesity, with particular emphasis on the members of the Bacteroidetes and Firmicutes phyla. They demonstrated that an obesityrelated microbiome has an apparent increased ability to harvest energy from the host's diet. Using deep tag-encoded sequencing, Larsen et $a l^{(10)}$ observed a significant reduction in the relative abundances of Firmicutes and Clostridia in adults with type 2 diabetes. Many diseases, including IBD, have been reported to be associated with an overall community dysbiosis rather than with changes in a single bacterial species ${ }^{(12)}$. For example, Dicksved et al. ${ }^{(13)}$ reported that patients with Crohn's disease have lower gut microbial diversity when compared with healthy individuals, with a lower relative abundance of Bacteroides uniformis and higher relative abundances of Bacteroides ovatus and Bacteroides vulgatus. Although most agree that the gut microbiota contributes to these disease states and others in some way, many contradictions exist in the literature and cause-and-effect relationships are rarely demonstrated. Therefore, more clinical studies are required to confirm these findings and identify methods by which diseases may be prevented or managed by dietary or pharmaceutical interventions.

Dietary changes have been shown to have significant effects on the human gut microbiota, indicating that diet is important for the development of the gut microbiome and that nutritional interventions may be useful for re-establishing a healthy microbiome. While some researchers have compared quite broad differences in diet across cultures in observational studies (e.g. macronutrient concentration and refined $v$. processed foods), others have designed prospective clinical studies to test specific dietary treatments. Although both experimental methods have been useful for advancing our knowledge regarding the microbiome in these early stages, carefully executed prospective double-blind, placebocontrolled studies that have accurate estimates of nutrient intake and that are designed to test specific dietary treatments will be most useful moving forward. 
De Filippo et al. ${ }^{(42)}$ compared the faecal microbiota of European children (European Union) with that of children from a rural African village of Burkina Faso. Not surprisingly, because these populations consume very different diets (complex, refined Western diet $v$. simplistic unrefined diet) and live in vastly different environments, large differences in the gut microbiota between the two groups were reported. In general, the microbiome of children from Burkina Faso was predominated by Bacteroidetes, with an abundance of bacteria belonging to the Prevotella and Xylanibacter genera, which possess a set of bacterial genes for cellulose and xylan hydrolysis, something that was completely absent in children from the European Union. Moreover, children from the European Union had a significantly higher abundance of Enterobacteriaceae (Shigella and Escherichia) than those from Burkina Faso. In another study, Wu et al. ${ }^{(43)}$ introduced the concept of 'enterotypes' that were associated with long-term diet. According to these researchers, subjects consuming a high-fat diet had a Bacteroides-dominated enterotype, while those consuming a high-carbohydrate diet had a Prevotella-dominated enterotype. The enterotype concept has been met with much criticism, and because many recent publications have demonstrated the impact of acute dietary changes, such a concept must be re-evaluated.

One of the best-studied areas has been that of dietary fibres, prebiotics and other non-digestible carbohydrates. In our laboratory, we evaluated the effects of two novel fibres, polydextrose (PDX) and soluble corn fibre (SCF), on the human gut microbiome using 16S rRNA gene amplicon-based 454-pyrosequencing $^{(44,45)}$. In a replicated $3 \times 3$ Latin square design using $21 \mathrm{~d}$ periods, twenty participants consumed three treatment snacks per $d$ that contained no supplemental fibre (no-fibre control), PDX $(21 \mathrm{~g} / \mathrm{d})$ or SCF $(21 \mathrm{~g} / \mathrm{d})$ during each period. Our data indicated a beneficial shift in the gut microbiome of adults consuming PDX and SCF, with greater abundances of faecal Clostridiaceae and Veillonellaceae, but a lower abundance of Eubacteriaceae as well as a greater abundance of $F$. prausnitzii being observed after fibre consumption. In addition to shifts in the microbiome, faecal ammonia, 4-methylphenol, indole and branched-chain fatty acid concentrations decreased when subjects consumed PDX and SCF than when they consumed the no-fibre control. Faecal acetate, propionate and butyrate concentrations were lower when subjects consumed PDX than when they consumed SCF and the no-fibre control ${ }^{(45)}$. These two studies established some interesting associations between microbiota and host physiological parameters that support the potential application of these novel fibres as prebiotics in human nutrition and health.

Walker et al. ${ }^{(30)}$ examined the influence of resistant starch on the faecal microbiome of overweight men using $16 \mathrm{~S}$ rRNA gene-based sequencing methods and proved that dietary non-digestible carbohydrates lead to marked changes in the gut microbiota. A total of fourteen overweight men participated in the study and consumed three controlled diets, including diets high in resistant starch (type III resistant starch) or NSP or a reduced carbohydrate weight-loss diet over 10 weeks. They reported that volunteers who consumed the resistant starch diet had increased abundances of
Ruminococcus bromii, Oscillibacter spp. and Eubacterium rectale compared with those who consumed the NSP diet. Davis et $a l .{ }^{(46)}$ used the pyrosequencing of $16 \mathrm{~S}$ rDNA tags to evaluate the impact of prebiotic galacto-oligosaccharide (GOS) on the faecal microbiota of healthy human subjects. They collected faecal samples from eighteen healthy adults during a 12-week feeding trial in which each subject consumed a GOS-containing product, with four dosages (0, $2 \cdot 5$, 5 and $10 \mathrm{~g}$ ) of GOS. GOS induced significant compositional alterations in the faecal microbiota, principally by increasing the abundance of Actinobacteria, specifically of several distinct lineages of Bifidobacterium. Interestingly, the responses to GOS and the magnitude of the responses varied between individuals, were reversible, and were in accordance with the dosage. Li et al. ${ }^{(47)}$ conducted a randomised, cross-over, controlled feeding study to determine whether cruciferous vegetable ( $14 \mathrm{~g} / \mathrm{kg}$ body weight per d; e.g. broccoli and cabbage) consumption altered the composition of faecal bacterial community. Using the terminal RFLP method, they showed that there was a significant difference in the faecal bacterial community after $14 \mathrm{~d}$ of consuming a cruciferous vegetablerich diet compared with a fruit- and vegetable-free basal diet. In addition, they also identified several putative gut bacterial species that were associated with the specific diets, including Eubacterium halli, Phascolarctobacterium faecium, Burkholderiales spp., Alistipes putredinis and Eggerthella spp., indicating that gut bacteria can be modified rapidly by dietary vegetable components. Unfortunately, none of these studies measured useful physiological parameters that could be linked to alterations in microbiota. More studies designed to identify diet-driven alterations in the gut microbiota, including controlled diet composition and dietary supplementation, are summarised in the review of Flint et al. ${ }^{(29)}$, and this review should be referred to for more in-depth information on the topic.

Although a paucity of publications focused on diet-induced gut microbiome changes in humans exists, it is clear that acute dietary changes alter the activity and composition of the human gut microbiome. Unfortunately, the majority of human microbiome studies carried out thus far have failed to measure physiological parameters relevant to the gut or overall host health. Because faecal DNA provides information only about potential functions of the microbiome, other measures of host physiology and health status are required to be studied. In addition, changes due to age, genetics, ethnicity and living environment cannot be ignored, and these may contribute to the alterations in gut microbiome in humans as well. Initial human microbiome studies have laid a foundation for future studies that may provide a better understanding of the functions and mechanisms of human gut microbes by which they influence human health.

\section{Canine and feline microbiomes}

Compared with humans and other livestock species, dogs and cats have evolved as carnivores and have a relatively simple GIT and do not rely on the microbiota for maintaining energy balance. Domestic cats, which are obligate carnivores, 
rely on high protein (HP)-containing animal tissues to meet their unique nutritional requirements in the wild and are metabolically adapted to a lower glucose utilisation and a higher protein metabolism ${ }^{(48)}$. Although it shares many anatomical and metabolic characteristics with the cat, the domestic dog is metabolically more omnivorous and can digest, absorb and metabolise a considerable amount of dietary carbohydrates. Although energy acquisition in dogs and cats does not rely heavily on microbial fermentation, a balanced and stable gut microbial community is critical for maintaining gastrointestinal health. Firmicutes, Bacteroidetes, Proteobacteria, Fusobacteria and Actinobacteria are the predominant microbial phyla in the canine and feline gut; however, their proportions vary among host species and individual studies reported in the literature. Variability may be due to animal (e.g. breed, diet and age), living environment or laboratory methodologies (e.g. sample collection and handling, DNA and RNA extraction methods, primers and sequencing methods).

Most of the canine microbiota studies using a comparative 16S rRNA gene approach have focused on the microbial community present in the colon or faecal samples. In one study, four major phylogenetic lineages were identified in the colon of six healthy hound dogs using 16S rRNA gene amplicon-based Sanger sequencing: Firmicutes (47.7\%); Proteobacteria (23.3\%); Fusobacteria (16.6\%); Bacteroidetes (12.4\%). Importantly, in this study, the microbial community in more proximal parts of the intestine was also evaluated and the diversity of the GIT microbial community from the duodenum to the colon was characterised. Clostridiales predominated in the duodenum (40\% of the clones) and jejunum (39\%) and were highly abundant in the ileum (25\%) and colon (26\%), while Fusobacteriales and Bacteroidales were the most abundant bacterial orders in the ileum (33\%) and colon (30\%). Enterobacteriales were more commonly observed in the small intestine than in the colon, and Lactobacillales occurred commonly in all parts of the intestine ${ }^{(49)}$.

In another study ${ }^{(50)}$, 16S rRNA gene amplicon-based 454-pyrosequencing was used for the first time to phylogenetically characterise the faecal microbiome of healthy dogs and Fusobacteria (23-40\% of the reads), Firmicutes (14-28\% of the reads), Bacteroidetes (31-34\% of the reads), Actinobacteria $(0 \cdot 8-1 \cdot 4 \%$ of the reads) and Proteobacteria $(5-7 \%$ of the reads) were found to be the co-dominant phyla in canine faeces. Using the same faecal samples from the above-mentioned study ${ }^{(50)}$ and shotgun 454-pyrosequencing, Swanson et al. ${ }^{(15)}$ reported the phylogeny and functional capacity of the microbiome of healthy dogs. Shotgun sequencing revealed Bacteroidetes (37-38\% of the sequences), Firmicutes (31-35\% of the sequences), Proteobacteria (13-15\% of the sequences), Fusobacteria (7-9\% of the sequences) and Actinobacteria ( $1 \%$ of the sequences) to be the dominant bacterial phyla in canine faeces. In addition to characterising the bacteria (98\% of all the sequences), the abundance of other micro-organisms, including eukaryota $(0 \cdot 4 \%)$, archaea (1\%) and viruses $(0 \cdot 3-0 \cdot 4 \%)$, was reported. The proportions (especially of Fusobacteria) were different from those reported by Middelbos et $a l .{ }^{(50)}$. Because the results were obtained from the analysis of the same faecal samples in both studies, the discrepancy must have been due to the differences in methodologies used, which include the biases involved with these assays (e.g. primer bias). Because shotgun sequencing was used, Swanson et al. ${ }^{(15)}$ also reported the predominant bacterial functional categories in the canine GIT: carbohydrates (12-13\% of the sequences); protein metabolism (8-9\%); DNA metabolism (7\%); cell wall and capsule (7-8\%); amino acids (AA) and derivatives (7\%); virulence (6-7\%); and cofactors, vitamins, prosthetic groups and pigments (6\%).

Foster et al. ${ }^{(51)}$ have recently characterised the fungal microbiome in canine faeces from both healthy dogs and dogs with acute diarrhoea. The dominant fungal phyla in the faeces of healthy dogs were Ascomycota (97.9\% of the sequences) and Basidiomycota (1.0\% of the sequences). However, no significant differences were observed in fungal composition between the healthy and diseased dogs. Hand et al. ${ }^{(52)}$ were the first to compare the faecal microbiota among a number of closely related dogs (e.g. breed and genetics) using $16 \mathrm{~S}$ rDNA gene amplicon-based Roche-454-pyrosequencing. They showed that although variability existed in prominent genera, genetically related dogs have a more similar faecal microbial composition when compared with unrelated dogs. In addition, Garcia-Mazcorro et al. ${ }^{(53)}$ described the temporal variability of the faecal microbiome in healthy dogs and the discrepancies in faecal microbial abundance data obtained using FISH and 454-pyrosequencing for analysis.

Similar to that in dogs, several technologies have been used to determine the phylogeny and functional capacity of the gut microbiome of healthy cats. Inness et al. ${ }^{(54)}$ were the first to use FISH to investigate gut bacterial populations in healthy cats and reported greater abundances of Bacteroides spp., Bifidobacterium spp., Clostridium histolyticum subgp., Lactobacillus-Enterococcus subgp. and Desulfovibrio spp. in feline faeces. Ritchie et al. ${ }^{(55)}$ described the diversity of GIT microbial communities in healthy cats using a traditional $16 \mathrm{~S}$ rRNA gene-based approach. They observed five predominant bacterial phyla: Firmicutes (68\%), followed by Proteobacteria (14\%), Bacteroidetes (10\%), Fusobacteria (5\%) and Actinobacteria (4\%). The majority of clones fell within the order Clostridiales (54\%), followed by Lactobacillales in the jejunum and Bacteroidales in the ileum and colon. Using nextgeneration 16S rRNA gene 454-pyrosequencing methods, Garcia-Mazcorro et al. ${ }^{(56)}$ reported the most abundant faecal bacterial phyla to be Firmicutes (92-95\%) and Actinobacteria (4-7\%), with less than $1 \%$ present being Proteobacteria, Bacteroidetes and Fusobacteria.

A recent study conducted in our laboratory ${ }^{(16)}$ using shotgun 454-pyrosequencing has evaluated the phylogeny and functional capacity of the faecal microbiome of healthy adult cats. In this study, the dominant bacterial phyla included Firmicutes (36-50\%) Bacteroidetes (24-36\%) and Proteobacteria (11-12\%). Bacteria (99\%) were the dominant micro-organisms in feline faeces, followed by eukaryota $(1 \%)$ and fungi $(0.02 \%)^{(57)}$. Barry et al. ${ }^{(16)}$ also reported the functional capacity of the feline faecal microbiome, with results being similar to that reported in dogs ${ }^{(15)}$. Carbohydrates (15\%), clustering-based subsystems (14\%), protein metabolism (8\%), AA and derivatives (8\%), cell wall and 
capsule (7\%), DNA metabolism (7\%), virulence (6\%), and cofactors, vitamins, prosthetic groups and pigments $(6 \%)$ were the predominant functional categories identified. Although the cat is an obligate carnivore, this study suggested that its gut microbiome (e.g. microbial phylogeny and gene content) is relatively similar to that of omnivores.

These recent studies suggest that the predominant phyla of the canine and feline gut microbiome are similar to those of humans and rodent models, both of which are also dominated by Firmicutes and Bacteroidetes. The key difference appears to be the predominance of Fusobacteria in the gut microbiome (intestines or faeces) in dogs and cats. Even though it is not the largest genus that inhabits the gut, it often represents $10 \%$ or more of the sequences. Factors affecting the human gut microbiota (e.g. age, genetics, ethnicity, diet, health status and living environment) probably also affect the gut microbiota of dogs and cats.

\section{Gut microbiota of dogs and cats under disease conditions}

Similar to that observed in humans, a disrupted gut microbial community may contribute to intestinal disorders in dogs and cats, not only due to the invasion of the enteropathogens in the GIT, but also due to the participation of the gut microbiota in many processes important to host metabolism (e.g. bile acid metabolism and fermentation and utilisation of non-digestible carbohydrates and proteins).

Bell et al. ${ }^{(58)}$ used the gel-based technique RFLP to evaluate changes in the faecal microbiota of dogs with diarrhoea and found Clostridium perfringens, Enterococcus faecalis and Enterococcus faecium populations to be present abundantly when compared with healthy dogs. Jia et al. ${ }^{(59)}$ used FISH to investigate the predominant faecal microbiota of dogs with chronic diarrhoea. Dogs with chronic diarrhoea were found to have a significantly higher abundance of Bacteroides, but lower Lactobacillus-Enterococcus counts when compared with healthy control dogs. The FISH technique was used in two studies to investigate alterations in the gut microbiota of cats with or without IBD. Inness et al. ${ }^{(54)}$ reported that total bacteria, Bifidobacterium spp. and Bacteroides spp. counts were significantly lower, whereas Desulfovibrio spp. (producers of toxic sulphides) counts were higher in cats with IBD when compared with healthy cats. Janeczko et al. ${ }^{(60)}$ reported that cats with IBD had increased counts of mucosa-associated Enterobacteriaceae in duodenal biopsies. However, with the traditional gel-based and quantitative PCR technologies used in these studies, only a limited number of gut bacteria could be quantified. The recent studies that have investigated alterations in the gut microbiota of diseased dogs using DNA sequencing methods are summarised in Table 1 . To our knowledge, highthroughput sequencing has not been used to compare the GIT microbiota of healthy cats with that of diseased cats.

Dogs with IBD have been shown to have a lower abundance of Bacteroidetes, but a higher abundance of Proteobacteria when compared with healthy dogs. For example, Chaban et al. ${ }^{(61)}$ used 454-pyrosequencing of the universal chaperonin-60 (cpn60) gene to analyse the faecal samples of dogs with IBD and reported a lower abundance of

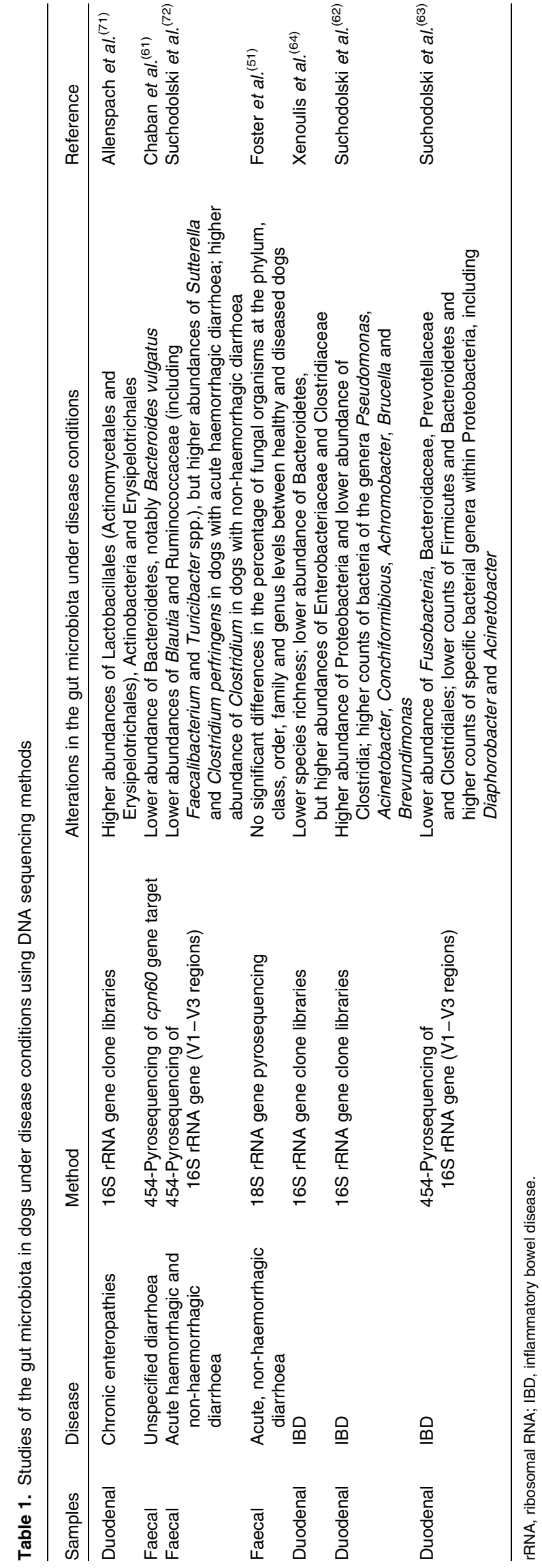


Bacteroidetes, notably B. vulgatus, compared with healthy controls. Suchodolski et al. ${ }^{(62,63)}$ used two different DNA sequencing methods to investigate the bacterial microbiota in the duodenal biopsies of dogs with IBD. Using 16S rRNA gene clone libraries, they reported that dogs with IBD had a significantly higher abundance of Proteobacteria and a lower abundance of Clostridia. Using 16S rRNA gene ampliconbased 454-pyrosequencing, they found a higher abundance of Proteobacteria (Diaphorobacter and Acinetobacter) and lower abundances of Clostridia, Fusobacteria, Bacteroidaceae and Prevotellaceae in dogs with IBD. In addition to a lower abundance of Bacteroidetes, Xenoulis et al. ${ }^{(64)}$ found higher abundances of Enterobacteriaceae and Clostridiaceae and a lower species richness in dogs with IBD. However, when comparing the entire microbial populations among these canine studies, it is clear that they are quite variable, which may be due to many factors, including methodology (e.g. DNA extraction method and primer bias), breed, age, diet and housing environment, as well as disease stage and medication use. Although many recent studies have mainly focused on the importance of maintaining a balanced intestinal microbial ecosystem, the cause-and-effect relationships between the gut microbiota and the host health conditions are still unclear. Because faecal 16S-based DNA provides information only about microbial identity, not about activity, microbial transcriptome and metabolome data along with more host physiological and histological data are needed to increase our understanding in this regard.

\section{Dietary effects on the gut microbiota in dogs and cats}

Commercial pet foods are highly variable with regard to their format, macronutrient composition, fibre content and source, and inclusion of 'functional' ingredients (e.g. prebiotics and probiotics, joint health modifiers, antioxidants and $n-3$ fatty acids). Many of these dietary factors have been investigated in canine and feline dietary studies, with emphasis on the gut microbial community in some cases. The most recent studies that have investigated the effects of diet on canine and feline gut microbiota using high-throughput DNA sequencing methods are summarised in Tables 2 and 3, respectively. All these studies indicate that dietary interventions may affect microbial composition and activity, with potential relevance to gut health. A few of these studies are briefly described below.

Typical pet foods can mainly be sorted into two categories: dry food and wet food. In addition to texture, pet foods differ greatly in terms of moisture, protein, fat and carbohydrate content. Bermingham et al. ${ }^{(65)}$ conducted a study to investigate the faecal microbial populations of cats fed either common conventional dry or wet foods using 16S rRNA gene amplicon-based 454-pyrosequencing. They demonstrated that a short-term dietary change in format led to large shifts in faecal bacterial populations. More specifically, cats fed the dry diet, which contained moderate protein and carbohydrate concentrations $(33 \%$ crude protein and $46 \%$ carbohydrate on DM basis (DMB)), had a higher abundance of Actinobacteria and lower abundances of Fusobacteria and Proteobacteria compared with those fed the wet diet containing HP and low carbohydrate concentrations ( $42 \%$ crude protein and $5 \%$ carbohydrate; DMB). In this study, however, shifts in bacterial populations could not be attributed to any specific nutrients because several items (e.g. dietary macronutrients and specific ingredients) differed between the diets.

The DNA sequencing technology was used in three recent studies to demonstrate the changes in faecal microbial populations in response to changes in dietary macronutrient composition in dogs and cats. Hang et al. ${ }^{(66)}$ reported that a HP diet (61\% of protein; DMB) favoured the growth of representatives of Fusobacteriales, whereas a high-carbohydrate diet (19\% of protein; DMB) favoured the growth of representatives of Erysipelotrichales in dogs using the faecal microbial DNA analysis by guanine-cytosine profiling $(\% \mathrm{G}+\mathrm{C})$. However, the method used in this study could not provide an overall picture of the canine faecal microbiota. Hooda et al. ${ }^{(67)}$ used 16S rRNA gene-based 454-pyrosequencing methods to evaluate the effects of dietary protein:carbohydrate ratio on the faecal microbiota of 8-16-week-old kittens. They reported large shifts in a high number of microbial taxa. At the phylum level, there was a greater abundance of Actinobacteria, but a lower abundance of Fusobacteria in kittens fed a moderateprotein, moderate-carbohydrate (MPMC; 34\% protein and $19 \%$ fat; DMB) diet compared with those fed a HP, lowcarbohydrate diet (53\% protein and $24 \%$ fat; DMB). Many microbial shifts were observed at the genus and species levels in this study. Using the same faecal samples from these kittens, Deusch et al. ${ }^{(68)}$ used Illumina shotgun sequencing to explore the functional capacity of the microbiome. They observed strong diet-related differences in pathways related to amino acid biosynthesis and metabolism. The microbiomes of kittens fed the MPMC diet had a greater abundance of genes related to pathways associated with the biosynthesis of amino acids, vitamins, fatty acids, and peptidoglycans; the glycolytic, TCA, and pentose phosphate pathways; oxidative phosphorylation; and the metabolism of purines, pyrimidines and sugars.

Many researchers have emphasised the beneficial effects of feeding non-digestible carbohydrates, prebiotics and/or probiotics to dogs and cats. While prebiotics are non-digestible food substances that increase the number or activity of specific beneficial bacteria already residing in the host, probiotics supply an exogenous source of beneficial live bacteria to the host. Prebiotic consumption often results in a greater production of fermentative end products such as SCFA in the colon that confer many benefits on the host. Probiotics may enhance intestinal health by displacing intestinal pathogens, producing antimicrobial substances or increasing immune responses. Synbiotics are preparations that contain a combination of prebiotics and probiotics. Using next-generation sequencing, researchers have been able to determine the ability of functional ingredients to change gut microbial ecology. Middelbos et al. ${ }^{(50)}$ investigated the effects of dietary inclusion of $7.5 \%$ beet pulp, a common fibre source in pet foods, on faecal microbial populations in dogs using 16S rRNA gene amplicon-based 454-pyrosequencing. Dietary inclusion of beet pulp was found to decrease the abundance of Fusobacteria, but increase the abundance of Firmicutes compared with the $0 \%$ supplemental fibre diet. Using a shotgun 
Table 2. Diet-related microbiome studies using DNA sequencing methods in dogs

\begin{tabular}{|c|c|c|c|c|}
\hline Samples & Dietary treatment & Method & Alterations in the gut microbiota & Reference \\
\hline Faecal & $\begin{array}{l}\text { HC ( } 61 \% \text { of protein; DMB) dry kibble diet } v . \mathrm{HP} \\
\text { ( } 19 \% \text { of protein; DMB) dry kibble diet } v . \text { dry kibble } \\
\text { commercial (DC; } 23 \% \text { of } \\
\text { protein; DMB) diet }\end{array}$ & $\begin{array}{l}\text { Escherichia coli } 16 \mathrm{~S} \text { rRNA } \\
\text { gene-based Sanger } \\
\text { sequencing (positions } \\
8-27 \text { and } 1389-1405 \text { ) }\end{array}$ & $\begin{array}{l}\text { Sequence diversity was highest in dogs fed the DC diet. } \\
\text { The abundances of Clostridiales, Lactobacillales, } \\
\text { Coriobacteriales and Bacteroidales were increased } \\
\text { in dogs fed the DC diet, while Lactobacillales and } \\
\text { Bacteroidales were not detected in dogs fed the HP } \\
\text { and HC diets. The HP and HC diets also decreased the } \\
\text { abundances of members of the Lachnospiraceae family. } \\
\text { The HC diet favoured the growth of representatives of } \\
\text { Erysipelotrichales, while the HP diet favoured that of } \\
\text { representatives of Fusobacteriales }\end{array}$ & Hang et al. ${ }^{(66)}$ \\
\hline Faecal & Control dry kibble diet with 0 v. $7.5 \%$ beet pulp & $\begin{array}{l}\text { 454-Pyrosequencing of } 16 \mathrm{~S} \text { rRNA } \\
\text { gene (V3 region) }\end{array}$ & $\begin{array}{l}\text { The beet pulp diet decreased the abundances of } \\
\text { Fusobacteria and Actinobacteria, but increased that of } \\
\text { Firmicutes. The abundance of Clostridia was increased } \\
\text { and complemented by a decline in that of Erysipelotrichi } \\
\text { when dogs were switched to the beet pulp diet }\end{array}$ & Middelbos et al. ${ }^{(50)}$ \\
\hline $\begin{array}{l}\text { Faecal (pooled } \\
\text { samples) }\end{array}$ & Control dry kibble diet with 0 v. $7.5 \%$ beet pulp & Shotgun 454-pyrosequencing & $\begin{array}{l}\text { Control dogs had greater proportions of Bacteroidetes, } \\
\text { Fusobacteria and Proteobacteria, whereas those fed the } \\
\text { beet pulp diet had greater proportions of the } \\
\text { Bacteroidetes/Chlorobi group and Firmicutes. } \\
\text { Three fungal phylotypes (Gibberella zeae PH-1, } \\
\text { Neurospora crassa and Saccharomyces cerevisiae) } \\
\text { were present in controls, but none in those fed the } \\
\text { beet pulp diet. The beet pulp diet did not greatly alter } \\
\text { the gene sequence number of any KEGG } \\
\text { functional categories }\end{array}$ & Swanson et al..$^{(15)}$ \\
\hline Faecal & Dry kibble diet with 0 v. $25 \%$ cooked navy beans & $\begin{array}{l}\text { 454-Pyrosequencing of } 16 \mathrm{~S} \\
\text { rRNA gene (V4-V6 regions) }\end{array}$ & $\begin{array}{l}\text { No significant diet-induced differences were } \\
\text { observed in the microbial populations }\end{array}$ & Kerr et al. ${ }^{(73)}$ \\
\hline Faecal & $\begin{array}{l}\text { Six raw meat-based diets: (1) beef control; } \\
\text { (2) beef }+1.4 \% \text { inulin; (3) beef }+1.4 \% \text { YCW; } \\
\text { (4) chicken control; (5) chicken }+1.4 \% \text { inulin; } \\
\text { (6) chicken }+1.4 \% \text { YCW }\end{array}$ & $\begin{array}{l}\text { 454-Pyrosequencing of } 16 \mathrm{~S} \\
\text { rRNA gene (V4-V6 regions) }\end{array}$ & $\begin{array}{l}\text { Beef-based diets increased the abundance of Escherichia, } \\
\text { but decreased that of Anaerobiospirillum compared with } \\
\text { chicken-based diets. Inulin decreased the abundances of } \\
\text { Enterobacteriaceae and Megamonas, but increased that of } \\
\text { Lactobacillus compared with the control. Inulin also } \\
\text { decreased the abundance of Escherichia compared } \\
\text { with YCW. YCW increased the abundance of } \\
\text { Bifidobacterium compared with inulin and the control }\end{array}$ & Beloshapka et al. ${ }^{(74)}$ \\
\hline Faecal & $\begin{array}{l}\text { Synbiotic containing a mixture of seven probiotic } \\
\text { strains and a blend of fructo-oligosaccharides } \\
\text { and arabinogalactans }\end{array}$ & $\begin{array}{l}\text { Escherichia coli } 16 \mathrm{~S} \text { rRNA } \\
\text { gene 454-pyrosequencing }\end{array}$ & $\begin{array}{l}\text { Abundances of Enterococcus and Streptococcus spp. } \\
\text { increased during at least one time point during } \\
\text { administration and returned to baseline values after } \\
\text { discontinuation of treatment (determined using qPCR). } \\
\text { However, the synbiotic did not significantly alter the } \\
\text { composition of faecal bacterial phyla }\end{array}$ & $\begin{array}{l}\text { Garcia-Mazcorro } \\
\quad \text { et al. }{ }^{(56)}\end{array}$ \\
\hline
\end{tabular}

HC, high carbohydrate; DMB, DM basis; HP, high protein; rRNA, ribosomal RNA; qPCR, quantitative PCR; DC, dry kibble commercial; YCW, yeast cell wall extract. 
Table 3. Diet-related microbiome studies using DNA sequencing methods in cats

\begin{tabular}{|c|c|c|c|c|}
\hline Samples & Dietary treatment & Method & Alterations in the gut microbiota & Reference \\
\hline Faecal & $\begin{array}{l}\text { Dry ( } 33 \% \text { protein, } 11 \% \text { fat and } 46 \% \text { carbohydrate; DMB) } \\
\text { V. wet ( } 42 \% \text { protein, } 42 \% \text { fat and } 5 \% \text { carbohydrate; } \\
\text { DMB) diets }\end{array}$ & $\begin{array}{l}\text { 454-Pyrosequencing of } \\
\text { 16S rRNA gene }\end{array}$ & $\begin{array}{l}\text { The dry diet increased the abundance of Actinobacteria and decreased } \\
\text { those of Fusobacteria and Proteobacteria compared with the wet diet. } \\
\text { The dry diet increased the abundances of Lactobacillus, Megasphaera } \\
\text { and Olsenella and decreased that of Bacteroides and Blautia }\end{array}$ & $\begin{array}{l}\text { Bermingham } \\
\text { et al. }{ }^{(65)}\end{array}$ \\
\hline Faecal & $\begin{array}{l}\text { HPLC (53\% protein, } 24 \% \text { fat and } 31 \% \text { carbohydrate; DMB) } \\
\text { dry kibble diet } v \text {. MPMC (34\% protein, } 9 \% \text { fat and } \\
11 \% \text { carbohydrate; DMB) dry kibble diet }\end{array}$ & $\begin{array}{l}\text { 454-Pyrosequencing of } \\
\text { 16S rRNA gene } \\
\text { (V4-V6 regions) }\end{array}$ & $\begin{array}{l}\text { The abundance of Actinobacteria was increased and that of Fusobacteria } \\
\text { was decreased in the MPMC group. The abundances of Clostridium, } \\
\text { Faecalibacterium, Ruminococcus, Blautia and Eubacterium were higher } \\
\text { in the HPLC group, while those of Dialister, Acidaminococcus, } \\
\text { Bifidobacterium, Megasphaera and Mitsuokella were higher } \\
\text { in the MPMC group }\end{array}$ & Hooda et al. ${ }^{(67)}$ \\
\hline Faecal & $\begin{array}{l}\text { HPLC (53\% protein, } 24 \% \text { fat and } 31 \% \text { carbohydrate; DMB) } \\
\text { dry kibble diet } v \text {. MPMC ( } 34 \% \text { protein, } 9 \% \text { fat and } \\
11 \% \text { carbohydrate; DMB) dry kibble diets }\end{array}$ & Shotgun sequencing & $\begin{array}{l}\text { The abundances of Firmicutes and Actinobacteria were lower, but those } \\
\text { of Fusobacteria and Proteobacteria were higher in the HPLC group. } \\
\text { Fusobacterium, Clostridium, Eubacterium, Ruminococcus, } \\
\text { Bacteroides and Desulfovibrio were more abundant in the HPLC group. } \\
\text { Megasphaera, Bifidobacterium, Acidaminococcus, Selenomonas, and } \\
\text { Prevotella were more abundant in the MPMC group. Pathways related } \\
\text { to amino acid biosynthesis and metabolism exhibited strongest } \\
\text { diet-related differences }\end{array}$ & Deusch et al. ${ }^{(68)}$ \\
\hline Faecal & $\begin{array}{l}\text { High-protein:fat:carbohydrate wet diet }(45: 37: 2 \% \text { DM) } \\
\text { v. moderate-protein:fat:carbohydrate dry diet } \\
\text { (35:20:28\%; DMB) }\end{array}$ & $\begin{array}{l}\text { 454-Pyrosequencing of } \\
\text { 16S rRNA gene }\end{array}$ & $\begin{array}{l}\text { Increased abundances of Firmicutes and Actinobacteria and a } \\
\text { decreased abundance of Fusobacteria were observed in kittens } \\
\text { fed the dry diet compared with those fed the wet diet after weaning }\end{array}$ & $\begin{array}{l}\text { Bermingham } \\
\text { et al. }{ }^{(75)}\end{array}$ \\
\hline Faecal & Two commercial canned therapeutic diets ( $\mathrm{X}$ and $\mathrm{Y})$ & $\begin{array}{l}\text { 454-Pyrosequencing of } \\
\text { 16S rRNA gene } \\
\text { (V1-V2 regions) }\end{array}$ & $\begin{array}{l}\text { Both therapeutic diets attenuated the clinical symptoms of diarrhoea } \\
\text { and altered the intestinal bacterial populations. Coriobacteriaceae } \\
\text { Slackia spp., Campylobacter upsaliensis, Enterobacteriaceae } \\
\text { Raoultella spp., Coriobacteriaceae Collinsella spp., and bacteria } \\
\text { of unidentified genera within the families of Clostridiales } \\
\text { Lachnospiraceae and Aeromonadales Succinivibrionacease } \\
\text { exhibited strong correlations with faecal score }\end{array}$ & Ramadan et al. ${ }^{(69)}$ \\
\hline Faecal & $\begin{array}{l}\text { Dry kibble diets containing } 4 \% \text { cellulose, } \\
4 \% \text { FOS or } 4 \% \text { pectin }\end{array}$ & Shotgun sequencing & $\begin{array}{l}\text { The pectin diet increased the abundances of Firmicutes, } \\
\text { Chlorobi, Elusimicrobia, Proteobacteria and total bacteria. } \\
\text { The FOS diet increased the abundances of Actinobacteria and } \\
\text { gene content related to amino acid metabolism compared with } \\
\text { the pectin diet. Faecal bacteria N metabolism was increased } \\
\text { in the pectin-fed cats. Carbohydrate-active enzymes revealed } \\
\text { increases in the abundance of several glycoside hydrolases, } \\
\text { glycosyl transferases and carbohydrate-binding molecules } \\
\text { with FOS and pectin consumption }\end{array}$ & Barry et al. ${ }^{(16)}$ \\
\hline
\end{tabular}

DMB, DM basis; rRNA, ribosomal RNA; HPLC, high-protein, low-carbohydrate; MPMC, moderate-protein, moderate-carbohydrate; FOS, fructo-oligosaccharides. 
metagenomic analysis, Swanson et al. ${ }^{(15)}$ reported that the inclusion of $7.5 \%$ beet pulp in a low-fibre dog food increased the proportions of the Bacteroidetes/Chlorobi group and Firmicutes, whereas the inclusion of $7.5 \%$ beet pulp did not greatly alter the gene sequence number of any Kyoto Encyclopedia of Genes and Genomes (KEGG) functional categories. Barry et al. ${ }^{(16)}$ used shotgun 454-pyrosequencing to evaluate the effects of a diet containing dietary fructooligosaccharides (FOS; 4\%) or pectin (4\%) compared with a cellulose $(4 \%)$ control diet in cats. They reported that the FOS diet increased the faecal proportion of Actinobacteria, while the pectin diets increased the proportions of Firmicutes and total bacteria. They also reported that the FOS diet increased the abundance of genes associated with amino acid metabolism, while the pectin diet increased the abundance of genes associated with $\mathrm{N}$ metabolism. When specific carbohydrate-active enzymes were analysed using the Carbohydrate-Active enZYmes Database (http://www.cazy.org/), consumption of both FOS and pectin was found to lead to modifications in the abundance of several glycoside hydrolases, glycosyl transferases and carbohydrate-binding molecules.

Garcia-Mazcorro et al. ${ }^{(56)}$ evaluated a commercially available multi-species synbiotic formulation designed for use in cats and dogs. The blend contained FOS, arabinogalactans, and a total of five billion $\left(5 \times 10^{9}\right)$ colony forming unit (CFU) of a mixture of seven bacterial species (E. faecium, Streptococcus salivarius ssp. thermophilus, Bifidobacterium longum, Lactobacillus acidopbilus, Lactobacillus casei ssp. rhamnosus, Lactobacillus plantarum, and Lactobacillus delbrueckii ssp. bulgaricus). In this study, changes in the faecal microbiota were analysed using denaturing gradient gel electrophoresis, 16S rRNA gene libraries, quantitative PCR and 16S rRNA gene 454-pyrosequencing. Even though Garcia-Mazcorro et al. ${ }^{(56)}$ observed increased Enterococcus and Streptococcus spp. counts during at least one time point during administration, these counts were found to return to baseline values after discontinuation of synbiotic treatment when using the quantitative PCR method. When using $16 \mathrm{~S}$ rRNA gene amplicon-based 454-pyrosequencing, the synbiotic formulation was found to not lead to significant changes in the proportions of the most abundant faecal bacterial phyla.

Ramadan et al. ${ }^{(69)}$ have recently evaluated two commercial therapeutic diets in cats with chronic diarrhoea and reported changes in the faecal microbiota using 16S rRNA gene amplicon-based 454-pyrosequencing. Interestingly, using orthogonal partial least-square-based statistical analysis, strong correlations between microbiota and faecal score were observed, including bacteria within the Coriobacteriaceae and Enterobacteriaceae families and unidentified genera within the order Clostridiales, which may indicate their link to gut health. Due to the lack of a control group (healthy cats) and testing of complicated diets containing many different ingredients, however, it is not possible to identify specific cause-and-effect relationships.

Overall, these studies demonstrate that dietary intake may modulate the composition or activity of the gut microbiota in dogs and cats as it does in humans. However, it should be noted that most studies have focused only on faecal samples, have only evaluated genomic DNA, and have been carried out in healthy adult populations of laboratory animals and that specific microbial shifts are not universal and are often affected by the baseline composition of the host's gut microbiota. Therefore, more research is needed in this area to gain a better understanding of dietary effects on the gut microbial community and how they may affect host health.

\section{Future opportunities and challenges}

Given their place in people's lives, companion animals share living environment, food and microbial populations with people $^{(17)}$ and serve as potential vectors for pathogen exposure. Moreover, cats, in particular, are obligate carnivores, evolving to thrive on HP, high-fat diets, potentially serving as a valuable comparative model for studying gut microbiome-host metabolism interactions. Therefore, the study of canine and feline microbiomes may not only bring benefits to the health of companion animals, but also to pet owners. The information (e.g. databases and bioinformatic tools) and experience gained from the HMP have provided a good foundation for companion animal microbiome studies. Although metabolic differences exist among host species, research carried out in human subjects and other animal models may be applied to dogs and cats in many instances. Because there is often a great lack of funding sources available and the research community focused on the canine or feline microbiome is small, progress in this area has been hindered. Thus, compared with the progress made in human research, there are still many questions that remain to be answered. As usual, industry sponsors and dog- and catspecific research foundations will be critically important in moving this field forward.

Recent studies have provided a foundation from which to work, but most of them have not reported enough data to be useful from a practical standpoint. With regard to disease, studies have primarily focused on specific enteropathogens associated with GI diseases rather than on the cause-andeffect relationships between microbiota and host health conditions. In some populations, for example, enteropathogens (e.g. Escherichia coli, Salmonella, Clostridium difficile, C. perfringens and Campylobacter jejuni) are present in both diseased and healthy animals ${ }^{(70)}$. Future research needs to establish associations between microbial and host metabolism or physiology. Another issue is that the vast majority of dog- and cat-specific studies carried out thus far have focused on faecal DNA, without any indicators of microbial activity, measures of host health, and accurate dietary information. To improve the field, it will be essential to understand not only the phylogenetic structure and functional capacity of the microbiome, but the activity and responsiveness of the microbiota, identifying relevant microbe-microbe and microbe-host relationships and mechanisms by which microbes affect host GI and metabolic diseases. Finally, it will be important to determine whether and how antibiotics and other drug therapies, breed and disease affect or are affected by the gut microbiome and how this information may be used to improve diets, identify disease biomarkers and develop targeted disease therapies. 


\section{References}

1. Rigottier-Gois L, Bourhis AG, Gramet G, et al. (2003) Fluorescent hybridisation combined with flow cytometry and hybridisation of total RNA to analyse the composition of microbial communities in human faeces using 16S rRNA probes. FEMS Microbiol Ecol 43, 237-245.

2. Pinkel D, Straume T \& Gray JW (1986) Cytogenetic analysis using quantitative, high-sensitivity, fluorescence hybridization. Proc Natl Acad Sci U S A 83, 2934-2938.

3. Liu WT, Marsh TL, Cheng H, et al. (1997) Characterization of microbial diversity by determining terminal restriction fragment length polymorphisms of genes encoding 16S rRNA. Appl Environ Microbiol 63, 4516-4522.

4. Muyzer G, de Waal EC \& Uitterlinden AG (1993) Profiling of complex microbial populations by denaturing gradient gel electrophoresis analysis of polymerase chain reaction-amplified genes coding for 16S rRNA. Appl Environ Microbiol 59 $695-700$

5. Zoetendal EG, Akkermans AD \& De Vos WM (1998) Temperature gradient gel electrophoresis analysis of $16 \mathrm{~S}$ rRNA from human fecal samples reveals stable and host-specific communities of active bacteria. Appl Environ Microbiol 64, 3854-3859.

6. Weinstock GM (2012) Genomic approaches to studying the human microbiota. Nature 489, 250-256.

7. Kozich JJ, Westcott SL, Baxter NT, et al. (2013) Development of a dual-index sequencing strategy and curation pipeline for analyzing amplicon sequence data on the MiSeq Illumina sequencing platform. Appl Environ Microbiol 79, 5112-5120.

8. Turnbaugh PJ, Ley RE, Mahowald MA, et al. (2006) An obesity-associated gut microbiome with increased capacity for energy harvest. Nature 444, 1027-1031.

9. Ley RE (2010) Obesity and the human microbiome. Curr Opin Gastroenterol 26, 5-11.

10. Larsen N, Vogensen FK, van den Berg FW, et al. (2010) Gut microbiota in human adults with type 2 diabetes differs from non-diabetic adults. PLOS ONE 5, e9085.

11. Brown CT, Davis-Richardson AG, Giongo A, et al. (2011) Gut microbiome metagenomics analysis suggests a functional model for the development of autoimmunity for type 1 diabetes. PLOS ONE 6, e25792.

12. Spor A, Koren O \& Ley R (2011) Unravelling the effects of the environment and host genotype on the gut microbiome. Nat Rev Microbiol 9, 279-290.

13. Dicksved J, Halfvarson J, Rosenquist M, et al. (2008) Molecular analysis of the gut microbiota of identical twins with Crohn's disease. ISME J 2, 716-727.

14. Round JL \& Mazmanian SK (2009) The gut microbiota shapes intestinal immune responses during health and disease. Nat Rev Immunol 9, 313-323.

15. Swanson KS, Dowd SE, Suchodolski JS, et al. (2011) Phylogenetic and gene-centric metagenomics of the canine intestinal microbiome reveals similarities with humans and mice. ISME J 5, 639-649.

16. Barry KA, Middelbos IS, Vester Boler BM, et al. (2012) Effects of dietary fiber on the feline gastrointestinal metagenome. J Proteome Res 11, 5924-5933.

17. Song SJ, Lauber C, Costello EK, et al. (2013) Cohabiting family members share microbiota with one another and with their dogs. Elife 2, e00458.

18. Leener ED, Decostere A, De Graef EM, et al. (2005) Presence and mechanism of antimicrobial resistance among enterococci from cats and dogs. Microb Drug Resist 11, 395-403.
19. Gill SR, Pop M, Deboy RT, et al. (2006) Metagenomic analysis of the human distal gut microbiome. Science 312, $1355-1359$.

20. Gaboriau-Routhiau V, Rakotobe S, Lecuyer E, et al. (2009) The key role of segmented filamentous bacteria in the coordinated maturation of gut helper T cell responses. Immunity 31, 677-689.

21. Chow J, Lee SM, Shen Y, et al. (2010) Host-bacterial symbiosis in health and disease. Adv Immunol 107, 243-274.

22. Peterson J, Garges S, Giovanni M, et al. (2009) The NIH Human Microbiome Project. Genome Res 19, 2317-2323.

23. The Human Microbiome Project Consortium (2012) A framework for human microbiome research. Nature $\mathbf{4 8 6}$, 215-221.

24. The Human Microbiome Project Consortium (2012) Structure, function and diversity of the healthy human microbiome. Nature 486, 207-214.

25. Zeeuwen PL, Kleerebezem M, Timmerman HM, et al. (2013) Microbiome and skin diseases. Curr Opin Allergy Clin Immunol 13, 514-520.

26. Chen YE \& Tsao H (2013) The skin microbiome: current perspectives and future challenges. J Am Acad Dermatol 69, $143-155$.

27. Wade WG (2013) The oral microbiome in health and disease. Pharmacol Res 69, 137-143.

28. Nasidze I, Li J, Quinque D, et al. (2009) Global diversity in the human salivary microbiome. Genome Res 19, 636-643.

29. Flint HJ, Scott KP, Louis P, et al. (2012) The role of the gut microbiota in nutrition and health. Nat Rev Gastroenterol Hepatol 9, 577-589.

30. Walker AW, Ince J, Duncan SH, et al. (2011) Dominant and diet-responsive groups of bacteria within the human colonic microbiota. ISME J 5, 220-230.

31. Zoetendal EG, Rajilic-Stojanovic M \& de Vos WM (2008) High-throughput diversity and functionality analysis of the gastrointestinal tract microbiota. Gut 57, 1605-1615.

32. Tap J, Mondot S, Levenez F, et al. (2009) Towards the human intestinal microbiota phylogenetic core. Environ Microbiol 11, 2574-2584.

33. Arumugam M, Raes J, Pelletier E, et al. (2011) Enterotypes of the human gut microbiome. Nature 473, 174-180.

34. Nicholson JK, Holmes E, Kinross J, et al. (2012) Host-gut microbiota metabolic interactions. Science 336, 1262-1267.

35. Wong JM, de Souza R, Kendall CW, et al. (2006) Colonic health: fermentation and short chain fatty acids. $J$ Clin Gastroenterol 40, 235-243.

36. Samuel BS, Shaito A, Motoike T, et al. (2008) Effects of the gut microbiota on host adiposity are modulated by the short-chain fatty-acid binding $G$ protein-coupled receptor, Gpr41. Proc Natl Acad Sci U S A 105, 16767-16772.

37. Ridlon JM, Kang DJ \& Hylemon PB (2006) Bile salt biotransformations by human intestinal bacteria. J Lipid Res $\mathbf{4 7}$, 241-259.

38. Swann JR, Want EJ, Geier FM, et al. (2011) Systemic gut microbial modulation of bile acid metabolism in host tissue compartments. Proc Natl Acad Sci US A 108, Suppl. 1, 4523-4530.

39. Wang Z, Klipfell E, Bennett BJ, et al. (2011) Gut flora metabolism of phosphatidylcholine promotes cardiovascular disease. Nature $\mathbf{4 7 2}, 57-63$.

40. Martin FP, Sprenger N, Montoliu I, et al. (2010) Dietary modulation of gut functional ecology studied by fecal metabolomics. J Proteome Res 9, 5284-5295.

41. Qin J, Li R, Raes J, et al. (2010) A human gut microbial gene catalogue established by metagenomic sequencing. Nature 464, 59-65. 
42. De Filippo C, Cavalieri D, Di Paola M, et al. (2010) Impact of diet in shaping gut microbiota revealed by a comparative study in children from Europe and rural Africa. Proc Natl Acad Sci U S A 107, 14691-14696.

43. Wu GD, Chen J, Hoffmann C, et al. (2011) Linking long-term dietary patterns with gut microbial enterotypes. Science 334, 105-108.

44. Hooda S, Boler BM, Serao MC, et al. (2012) 454 pyrosequencing reveals a shift in fecal microbiota of healthy adult men consuming polydextrose or soluble corn fiber. $J$ Nutr 142, $1259-1265$

45. Boler BM, Serao MC, Bauer LL, et al. (2011) Digestive physiological outcomes related to polydextrose and soluble maize fibre consumption by healthy adult men. $\mathrm{Br} J$ Nutr 106, 1864-1871.

46. Davis LMG, Martinez I, Walter J, et al. (2011) Barcoded pyrosequencing reveals that consumption of galactooligosaccharides results in a highly specific bifidogenic response in humans. PLOS ONE 6, e25200.

47. Li F, Hullar MA, Schwarz Y, et al. (2009) Human gut bacterial communities are altered by addition of cruciferous vegetables to a controlled fruit- and vegetable-free diet. $J \mathrm{Nutr}$ 139, 1685-1691.

48. MacDonald ML, Rogers QR \& Morris JG (1984) Nutrition of the domestic cat, a mammalian carnivore. Annu Rev Nutr 4, 521-562.

49. Suchodolski JS, Camacho J \& Steiner JM (2008) Analysis of bacterial diversity in the canine duodenum, jejunum, ileum, and colon by comparative 16S rRNA gene analysis. FEMS Microbiol Ecol 66, 567-578.

50. Middelbos IS, Vester Boler BM, Qu A, et al. (2010) Phylogenetic characterization of fecal microbial communities of dogs fed diets with or without supplemental dietary fiber using 454 pyrosequencing. PLOS ONE 5, e9768.

51. Foster ML, Dowd SE, Stephenson C, et al. (2013) Characterization of the fungal microbiome (mycobiome) in fecal samples from dogs. Vet Med Int 2013, e658373.

52. Hand D, Wallis C, Colyer A, et al. (2013) Pyrosequencing the canine faecal microbiota: breadth and depth of biodiversity. PLOS ONE 8, e53115.

53. Garcia-Mazcorro JF, Dowd SE, Poulsen J, et al. (2012) Abundance and short-term temporal variability of fecal microbiota in healthy dogs. Microbiologyopen 1, 340-347.

54. Inness VL, McCartney AL, Khoo C, et al. (2007) Molecular characterisation of the gut microflora of healthy and inflammatory bowel disease cats using fluorescence in situ hybridisation with special reference to Desulfovibrio spp. I Anim Physiol Anim Nutr (Berl) 91, 48-53.

55. Ritchie LE, Steiner JM \& Suchodolski JS (2008) Assessment of microbial diversity along the feline intestinal tract using $16 \mathrm{~S}$ rRNA gene analysis. FEMS Microbiol Ecol 66, 590-598.

56. Garcia-Mazcorro JF, Lanerie DJ, Dowd SE, et al. (2011) Effect of a multi-species synbiotic formulation on fecal bacterial microbiota of healthy cats and dogs as evaluated by pyrosequencing. FEMS Microbiol Ecol 78, 542-554.

57. Tun HM, Brar MS, Khin N, et al. (2012) Gene-centric metagenomics analysis of feline intestinal microbiome using 454 junior pyrosequencing. J Microbiol Methods 88, 369-376.

58. Bell JA, Kopper JJ, Turnbull JA, et al. (2008) Ecological characterization of the colonic microbiota of normal and diarrheic dogs. Interdiscip Perspect Infect Dis 2008, 149694.

59. Jia J, Frantz N, Khoo C, et al. (2010) Investigation of the faecal microbiota associated with canine chronic diarrhoea. FEMS Microbiol Ecol 71, 304-312.
60. Janeczko S, Atwater D, Bogel E, et al. (2008) The relationship of mucosal bacteria to duodenal histopathology, cytokine mRNA, and clinical disease activity in cats with inflammatory bowel disease. Vet Microbiol 128, 178-193.

61. Chaban B, Links MG \& Hill JE (2012) A molecular enrichment strategy based on cpn60 for detection of epsilonproteobacteria in the dog fecal microbiome. Microb Ecol 63, 348-357.

62. Suchodolski JS, Xenoulis PG, Paddock CG, et al. (2010) Molecular analysis of the bacterial microbiota in duodenal biopsies from dogs with idiopathic inflammatory bowel disease. Vet Microbiol 142, 394-400.

63. Suchodolski JS, Dowd SE, Wilke V, et al. (2012) 16S rRNA gene pyrosequencing reveals bacterial dysbiosis in the duodenum of dogs with idiopathic inflammatory bowel disease. PLOS ONE 7, e39333.

64. Xenoulis PG, Palculict B, Allenspach K, et al. (2008) Molecularphylogenetic characterization of microbial communities imbalances in the small intestine of dogs with inflammatory bowel disease. FEMS Microbiol Ecol 66, 579-589.

65. Bermingham EN, Young W, Kittelmann S, et al. (2013) Dietary format alters fecal bacterial populations in the domestic cat (Felis catus). Microbiologyopen 2, 173-181.

66. Hang I, Rinttila T, Zentek J, et al. (2012) Effect of high contents of dietary animal-derived protein or carbohydrates on canine faecal microbiota. BMC Vet Res $\mathbf{8}, 90$.

67. Hooda S, Boler BMV, Kerr KR, et al. (2013) The gut microbiome of kittens is affected by dietary protein:carbohydrate ratio and associated with blood metabolite and hormone concentrations. Br J Nutr 109, 1637-1646.

68. Deusch O, O'Flynn C, Colyer A, et al. (2014) Deep Illuminabased shotgun sequencing reveals dietary effects on the structure and function of the fecal microbiome of growing kittens. PLOS ONE 9, e101021.

69. Ramadan Z, Xu H, Laflamme D, et al. (2014) Fecal microbiota of cats with naturally occurring chronic diarrhea assessed using 16S rRNA gene 454-pyrosequencing before and after dietary treatment. $J$ Vet Intern Med 28, 59-65.

70. Marks SL \& Kather EJ (2003) Bacterial-associated diarrhea in the dog: a critical appraisal. Vet Clin North Am Small Anim Pract 33, 1029-1060.

71. Allenspach K, House A, Smith K, et al. (2010) Evaluation of mucosal bacteria and histopathology, clinical disease activity and expression of Toll-like receptors in German shepherd dogs with chronic enteropathies. Vet Microbiol 146, 326-335.

72. Suchodolski JS, Markel ME, Garcia-Mazcorro JF, et al. (2012) The fecal microbiome in dogs with acute diarrhea and idiopathic inflammatory bowel disease. PLOS ONE 7, e51907.

73. Kerr KR, Forster G, Dowd SE, et al. (2013) Effects of dietary cooked navy bean on the fecal microbiome of healthy companion dogs. PLOS ONE 8, e74998.

74. Beloshapka AN, Dowd SE, Suchodolski JS, et al. (2013) Fecal microbial communities of healthy adult dogs fed raw meat-based diets with or without inulin or yeast cell wall extracts as assessed by 454 pyrosequencing. FEMS Microbiol Ecol 84, 532-541.

75. Bermingham EN, Kittelmann S, Young W, et al. (2013) Post-weaning diet affects faecal microbial composition but not selected adipose gene expression in the cat (Felis catus). PLOS ONE 8, e80992. 